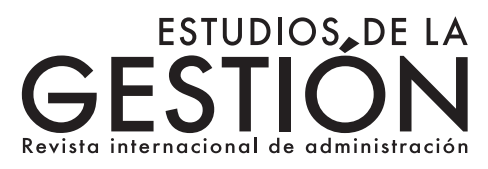

\title{
Los desafíos de la gestión por procesos en la era digital
}

The Challenges of Process Management in the Digital Age

Os desafios da gestão por processos na era digital

\author{
Juan Piñuela-Espín \\ Pontificia Universidad Católica del Ecuador (PUCE). Quito, Ecuador \\ juancarlospinuela@gmail.com \\ https://orcid.org/0000-0001-5649-3228 \\ Carla Quito-Godoy \\ Pontificia Universidad Católica del Ecuador (PUCE). Quito, Ecuador \\ carla.quito@gmail.com \\ https://orcid.org/0000-0002-5031-0854
}

DOI: https://doi.org/10.32719/25506641.2020.8.1

Recibido: 24 de enero de 2020 - Revisado: 31 de enero de 2020

Aceptado: 16 de marzo de 2020

Artículo de investigación

Licencia Creative Commons

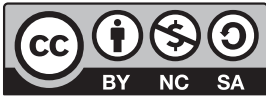




\section{Resumen}

La gestión por procesos es una herramienta fundamental para las organizaciones, es el elemento base para alcanzar objetivos de eficacia y eficiencia, además de ser un actor fundamental de la mejora continua y la productividad.

Este documento tiene como objetivo exponer diferentes posiciones sobre si el enfoque de procesos, como se conoció en la tercera revolución industrial, es vigente para resolver la complejidad de las organizaciones en la era digital, así como entender su relación con la creación de capacidades para atender los requisitos de clientes en un entorno de cambio dinámico.

A través del método deductivo y de forma indirecta se exploran los principales enfoques del management, el duro, que incluye la estructura organizativa, los procesos, sistemas, las mediciones y los riesgos, y el blando, centrado en el desarrollo de las personas. Estos dos enfoques han sido la respuesta tradicional ante la compleja operación de las organizaciones y mantienen relación directa con la gestión por procesos de la era industrial, por lo que es pertinente analizar si esa relación está vigente en la era digital.

Además, se identifican características que deben desarrollar los nuevos procesos para estar alineados con las transformaciones, en el contexto de la cuarta revolución industrial, como innovación, flexibilidad y adaptabilidad.

Palabras clave: Procesos, complejidad, capacidad, personas, cambio.

JEL: O33 Cambio tecnológico: opciones y consecuencias; difusión.

\section{Abstract}

Process management is a fundamental tool for the management of organizations, it is a basic element to achieve effectiveness objectives and efficiency, as well as a fundamental actor of continuous improvement and productivity.

This document aims to present a point of view on whether the process approach as it was known in the industrial revolution, is in force to solve the complexity of organizations in the digital age, its relationship with capacity building to meet the requirements of customers in a dynamic change environment.

Through the deductive method and indirectly the two main approaches to management are explored, the organizational structure, processes, systems, measurements and risk are on the hard line, and on the soft line there are relationships and people-focused development, these two approaches have been the traditional response to the complexity of the company and maintain a relationship with the management by processes in the industrial age, it is analyzed whether this relationship is in force in the digital age.

It is concluded by identifying certain characteristics that the new processes must develop in order to be aligned to the transformations in the context of the fourth industrial revolution such as innovation, flexibility and adaptability.

Keywords: Processes, complexity, capacity, people, change.

JEL: O33-Technological change: options and consequences; diffusion. 


\section{Resumo}

A gestão por processos é uma ferramenta fundamental para as organizações, um elemento base para se alcançarem objetivos de eficácia e eficiência, além de ser um item fundamental para a melhoria contínua e para a produtividade.

Este documento tem como objetivo expor alguns pontos de vista sobre se o enfoque de processos, tal como se conheceu na revolução industrial, é vigente para resolver a complexidade das organizações na era digital, assim como entender sua relação com a criação de capacidades para atender os requisitos de clientes em um entorno de mudanças dinâmicas.

Através do método dedutivo e de maneira indireta, exploram-se os dois principais enfoques do management, o hard, que inclui estrutura organizativa, processos, sistemas, medições e riscos, e o soft, centrado no desenvolvimento das pessoas. Esses dois enfoques têm sido a resposta tradicional frente à complexa operação das organizações e mantém relação direta com a gestão por processos da era industrial. Assim, é pertinente analisar se tal relação é vigente na era digital.

Conclui-se identificando certas características que devem desenvolver os novos processos para alinhar-se às transformações no contexto da quarta revolução industrial, como inovação, flexibilidade e adaptabilidade.

Palavras-chave: Processos, complexidade, capacidade, pessoas, mudança.

JEL: O33-Mudança tecnológica: escolhas e consequências; processos de difusão.

\section{Problemática y su contexto}

L

as organizaciones son estructuras de gestión que operan dentro de un contexto político, social, económico o legal, creadas para alcanzar objetivos; en sí, son un sistema compuesto por recursos, personas, procesos, infraestructura, tecnologías y otros elementos que contribuyen a cumplir su misión. La interacción e interrelación de las variables mencionadas crean un entorno complejo que desafía al management y que responde con metodologías, herramientas, modelos de gestión, desarrollo de personas o aplicación de buenas prácticas que permitan organizar y resolver la compleja realidad organizacional.

En la historia cercana de la Revolución Industrial, las organizaciones crearon y desarrollaron capacidades (Schwab 2019): la primera revolución utilizó agua y vapor para mecanizar la producción; la segunda utilizó la energía eléctrica para crear producción en masa y la tercera utilizó la electrónica y la tecnología de la información para automatizar la producción. 
La cuarta revolución se sustenta en un cambio digital que comenzó a registrarse desde mediados del siglo pasado y que tiene como particularidad la fusión de tecnologías que están borrando los límites entre las esferas físicas, digitales y biológicas y que tiene un efecto transversal a todas las disciplinas, las economías e industrias.

En las dos primeras revoluciones, la estructura organizativa (organigrama funcional) probablemente fue el principal elemento del management para resolver la compleja gestión. Entre la segunda y tercera revolución se desarrollaron, a la par y en sinergia, los modelos de calidad y la gestión por procesos.

Con la producción en masa inició el concepto de calidad en su primera etapa -la inspección-: la inclusión de prácticas de control estadístico, motivadas por Walter E. Shewhart, y de sistemas y procedimientos que impiden generar bienes defectuosos, promovida por Joseph M. Jurán; los sistemas de organización de la producción "Justo a Tiempo", de Taiichi Ohno; y el desarrollo de la ingeniería de procesos de Kauro Ishikawa. Estos modelos proyectaron las etapas de control y aseguramiento de la calidad. Un hito destacable es el ciclo PHVA (originario de Shewart e impulsado por Deming), como un proceso de mejora continua, el cual sembró el concepto de calidad total que ha conducido a los sistemas de gestión de la calidad que posteriormente fueron adoptados por la International Organization for Standardization (ISO). El management vio surgir al enfoque de procesos como elemento fundamental de la gestión en las organizaciones.

$\mathrm{Al}$ abordar los sistemas complejos, ${ }^{1}$ algunos científicos plantean la metáfora del caballo esférico - se deriva de bromas que se hacían los físicos teóricos- ${ }^{2}$ que pretende simplificar las variables de un sistema y aislarlo para facilitar su investigación. De acuerdo con la experiencia de los autores de este artículo, es probable que el enfoque de procesos represente la simplificación de la complejidad organizacional; para esta afirmación se referencia

1. Un sistema complejo tiene muchos elementos que se relacionan altamente e interconectados (Hilbert 2015).

2. Los físicos teóricos son aquellos investigadores que, en la aplicación de un estudio, optan por eliminar variables que permitan generar conclusiones iniciales para orientar mejor su investigación. 
el modelo de $5 \mathrm{~m}^{3}$ utilizado por Kauro Ishikawa, que en su famosa espina de pescado propone analizar todos los factores que involucran un proceso aislado en 5 variables principales, que se constituyeron en el principal motor para desarrollar la gestión en la tercera revolución industrial.

En el libro Gamestorming (Gray y Macanufo 2012) se expresa que las cadenas de procesos que transforman entradas en salidas ya no son suficientes en la era digital porque constriñen la complejidad. Al simplificar lo complicado está bien, pero cuando se intenta simplificar lo complejo se hace mal. Por ejemplo, los procesos burocráticos son complicados, pero se pueden reducir a un principio simple, entrada, proceso y salida; o puede ser que haya muchos, entrelazados y con retroactividad que influya entre ellos y que al ejecutarse varias veces se vean enredados. En cambio, en la complejidad existe interrelación, retroacción, incertidumbre, aleatoriedad y variabilidad, condiciones que se afectan unas a otras como en los procesos estocásticos o el clima (tiene muchas variables interrelacionadas). El problema es cuando se intenta reducir la complejidad a niveles comprensibles, lo que provoca simplificarla para que sea sencilla; el error es simplificar en exceso, evitando que los elementos complejos se puedan entender entre sí, pues implícitamente se renuncia a la complejidad y algo simple pudiera ser simplista (Ochoa 2018). Los modelos de cadenas de proceso utilizados en la era industrial también intentan explicar la organización en la era digital, simplificando la complejidad para hacerla comprensible; en ese ejercicio pudieran eliminar elementos de la complejidad subyacente (datos, relaciones entre aplicaciones informáticas, por ejemplo), que es necesario entender y gestionar.

La era digital plantea desafíos en la forma en que vivimos, trabajamos y nos relacionamos. Definitivamente, la velocidad, el alcance y el impacto de los cambios, que no son solo tecnológicos, se están ya experimentando. Entre las tecnologías que hacen posible la cuarta revolución industrial están principalmente la inteligencia artificial, el internet de las cosas, la robótica y el big data, por citar algunos; la robótica que ha sobrepasado su fase de prototipo en las fábricas pasa a ser parte de los sistemas de automatización utilizados en otros espacios laborales (Ochoa y Piñuela 2017). Las empresas

3. Materia prima, mano de obra, maquinaria, medioambiente y método. 
digitales, a más de tecnología, han desarrollado características particulares a su modelo de gestión, atributos que funcionan como un ecosistema que gira alrededor del cliente. Las características destacadas son:

1. Decisiones que se basan en datos omnipresentes y accesibles.

2. El cliente es lo primero, se enfocan en mejorar la experiencia en cada interacción.

3. Los datos están a disposición de los colaboradores, entonces las decisiones se toman rápidamente.

4. Toleran los errores, mantienen la filosofía de equivocarse rápido o en etapas tempranas. No penalizan a las personas que se equivocan.

5. Cultivan el talento, fomentan la autoorganización, el autocontrol y el aprendizaje continuo de las personas.

6. La flexibilidad es la norma, las estructuras rígidas o departamentales han dado paso a comunidades de desarrollo.

7. Lo cuestionan todo, no se conforman, cuestionan el statu quo de forma colaborativa.

8. Optimizan procesos, crean valor, se comparan, son productivos y simplifican de manera obsesiva.

La gestión por procesos ha sido una herramienta fundamental para las organizaciones, elemento base para alcanzar objetivos de eficacia y eficiencia, además de ser un actor fundamental de la mejora continua y la productividad.

En este contexto se plantea la siguiente pregunta: ¿el paradigma de la gestión por procesos vigente en la tercera revolución industrial puede aportar para resolver la complejidad de la gestión en la era digital?

\section{Metodología}

El objetivo de investigación consiste en exponer puntos de vista sobre si el enfoque de procesos, como se conoció principalmente en la tercera revolución industrial, es vigente para resolver la complejidad de las organizaciones en la era digital, y entender su relación con la creación de capacidades para atender los requisitos de clientes en un entorno de cambio dinámico. 
En primer lugar, se utiliza el método deductivo indirecto porque resulta del razonamiento que gira en torno a la gestión por procesos; se utiliza la exposición de características generales para presentar la evolución del enfoque de procesos y el impacto que tuvo como factor clave para resolver la complejidad del management organizacional a lo largo de las cuatro revoluciones industriales. En segundo lugar, se parte de la premisa universal y verdadera de que la gestión por procesos fue una herramienta fundamental para el management, particularmente en la tercera revolución industrial, y se analiza su vigencia para resolver la complejidad en la era digital o cuarta revolución industrial.

\section{Los principales enfoques del management}

Desde el siglo pasado y cada vez más frecuentemente, las organizaciones están experimentando una serie de cambios. Los clientes son cada vez más relevantes, los mercados son globales y permiten un sinnúmero de opciones, los nuevos modelos de negocio son obras de arte empresariales, la inclusión de tecnología disruptiva, la adopción de buenas prácticas de gestión y la aplicación de varias metodologías creadas para mejorar eficacia y eficiencia presentan un panorama de mayor apoyo a la gestión. La robotización, la automatización, el incremento en las competencias del personal y qué decir del know-how organizacional asociado a la disposición monumental de datos; con todo esto diríamos que tenemos más herramientas y recursos que nunca.

El managment organizacional ha tratado de resolver la complejidad organizacional a través de dos enfoques (Yves Morieux 2014, min. 2-12):

1. El enfoque duro: estructura organizacional, procesos, sistemas, métricas y control de riesgos a través de modelos predictivos que pretenden anticipar eventos futuros.

2. Enfoque blando: relaciones interpersonales, feeling, rasgos de personalidad y trabajo sobre la cultura organizacional.

Al evaluar organizaciones, sus problemas, necesidades o incremento de capacidades, se intenta resolver tomando uno, otro o una combinación de los dos enfoques descritos; sin embargo, no es tan simple. A continuación, se presentan dos ejemplos: 
a) Una empresa que decide ampliar operaciones en varias regiones y crea un nivel jerárquico adicional que genera un cambio en la estructura organizativa, agrega un sistema de control de gestión con indicadores. El cambio repercute en la gente y sus procesos; la adopción de métricas podría sesgar el enfoque únicamente en aquello que se puede medir (y aquello que no se mide pero es necesario puede quedarse fuera), es decir, que al gestionar alguna variable es evidente que hay efecto en otras variables organizativas.

b) Las empresas automotrices experimentaron varios cambios cuando se incorporó un nuevo requisito del cliente conocido como la extensión del tiempo de garantía, lo que significó la creación de canales de atención, centros de soporte, cambios en el propio producto y servicio, incremento de personal, probablemente incentivos de servicio y compensaciones, en definitiva, nuevos procesos, indicadores, variables adicionales para responder al nuevo requisito.

En los casos expuestos, es posible que los sistemas y estructura estuviesen dedicados al proceso como actor fundamental de la respuesta al cliente o la necesidad organizativa, y al incrementar capacidades organizacionales terminaban agregando más complejidad al sistema. Un paradigma dominante era el modelo predictivo, pues el énfasis estaba en una planificación detallada y a largo plazo, y la velocidad de cambio en la era industrial permitía proyecciones a cinco diez años.

Con frecuencia se escucha hablar del mundo VUCA, ${ }^{4}$ el cual ha hecho que en varios entornos empresariales se consolide la incapacidad para conceptualizar con precisión las posibles amenazas y oportunidades antes de que sea muy tarde. Está dinámica de cambios es tan vertiginosa que no solo amenaza a las organizaciones sino a los mismos profesionales, y es probable que los dos enfoques del management sean insuficientes en entornos de esta naturaleza.

4. VUCA: Volatility se asocia a la dinámica de los cambios y a la velocidad con que estos se producen, Uncertainty es el incremento de las situaciones imprevistas, Complexity son los problemas, conflictos que hay que resolver y no hay conexión entre causas y efectos, Ambiguity es la falta de claridad sobre el significado de cualquier hecho. 
En la era predigital la ventaja competitiva era eficiencia, optimización y economías de escala. Desarrollar capacidades organizacionales estaba asociado a la gestión por procesos y sus componentes.

En la era digital el éxito proviene de desarrollar capacidades tales como innovación, adaptabilidad, personalización y rapidez; la estrategia debe desglosarse en una cartera de pequeñas piezas de valor que puedan priorizarse; las cosas grandes llevan mucho tiempo, las pequeñas son más rápidas. Crear una cultura organizacional adaptativa es importante; la tecnología adecuadamente enfocada puede expandir capacidades para intentar captar la mayor cantidad de oportunidades (Highsmith y Robinson 2020).

La norma ISO9001 es un modelo de gestión que combina los dos enfoques del management; además, se sustenta en el enfoque de procesos y riesgos como elementos base para gestionar la calidad y la mejora continua. La última versión de la norma (ISO 2015) trajo cambios para fortalecer el vínculo con el entorno y establecer el contexto de la organización acorde con la política de la calidad y la estrategia. La norma menciona que cada organización deba determinar "los procesos necesarios" para el sistema de gestión de calidad; sin embargo, es común preguntarse si los procesos elegidos permiten crear capacidades que requiere la organización, sin complejizarla y en un modelo dinámico que pudiera enfrentar los desafíos de entornos VUCA.

La experiencia de los autores de este artículo en más de 80 proyectos en organizaciones del mercado ecuatoriano expone dos realidades diametralmente opuestas. Empresas que cuentan con un sistema de gestión de calidad (implementado y en varios casos certificado) mencionan una exagerada carga administrativa y documental creada para cumplir requisitos donde el sistema se activa previa la ejecución de auditorías o cuando es necesario mostrarse externamente. Otro grupo de empresas (menor que el primero) aseguran que el sistema de calidad ha impactado positivamente; la mejora continua se tradujo en resultados económicos, ahorros por disminución de reprocesos y mejora en ingresos por incremento en la satisfacción del cliente.

En los gráficos que se muestran a continuación se exponen los tipos de organizaciones donde los autores han desarrollado proyectos de consultoría; se identifican aquellas que contaban con un sistema de gestión y se exponen las que tenían modelos complicados. 


\section{Figura 1}

\section{Proyectos de consultoría por tipo de organización}
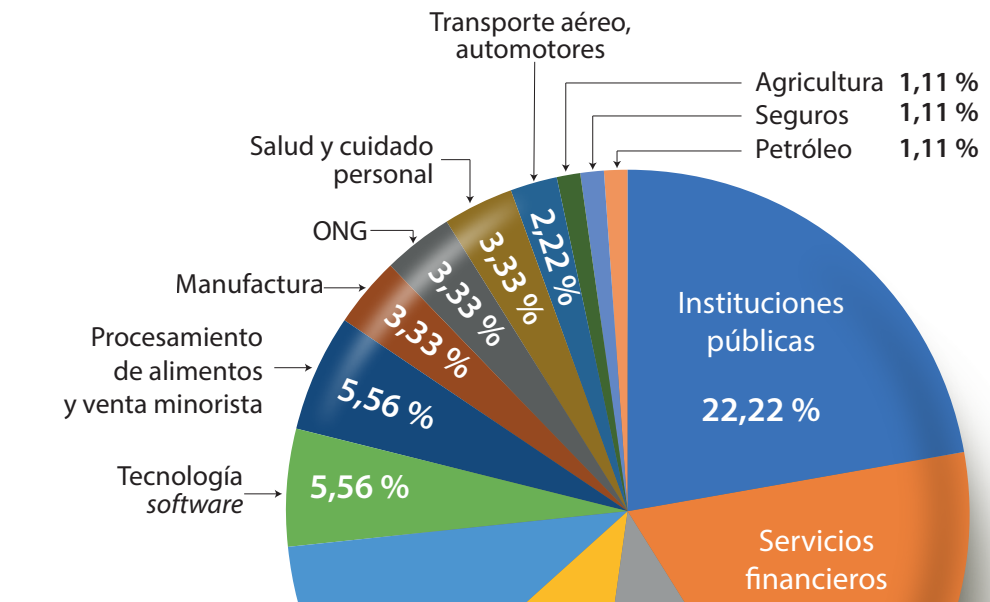

Telecomunicaciones $\longrightarrow \quad 10,00 \% \quad 18,89 \%$

\section{Educación Servicios} públicos

$11,11 \%$

\section{$11,11 \%$}

Fuente y elaboración propia.

Tabla 1

Proyectos de consultoría por tipo de organización

\begin{tabular}{|c|c|c|c|c|}
\hline \multirow{2}{*}{$\begin{array}{l}\text { Proyectos de consultoría por segmento de } \\
\text { empresa }\end{array}$} & \multicolumn{3}{|c|}{ Cuenta con sistema de gestión } & \multirow{2}{*}{$\begin{array}{c}\text { Porcentaje } \\
(\%)\end{array}$} \\
\hline & No & Sí & Total general & \\
\hline Instituciones públicas & 15 & 5 & 20 & 22,22 \\
\hline Servicios financieros & 9 & 8 & 17 & 18,89 \\
\hline Servicios públicos & 7 & 3 & 10 & 11,11 \\
\hline
\end{tabular}




\begin{tabular}{|l|c|c|c|c|}
\hline Educación & 6 & 4 & 10 & 11,11 \\
\hline Telecomunicaciones & 1 & 8 & 9 & 10,00 \\
\hline Tecnología-software & 5 & & 5 & $\mathbf{5 , 5 6}$ \\
\hline Procesamiento de alimentos y venta minorista & 5 & & 5 & $\mathbf{5 , 5 6}$ \\
\hline Manufactura & 2 & 1 & 3 & 3,33 \\
\hline ONG & 1 & 2 & 3 & 3,33 \\
\hline Salud y cuidado personal & & 3 & 3 & 3,33 \\
\hline Transporte aéreo, automotores & 1 & 1 & 2 & $\mathbf{2 , 2 2}$ \\
\hline Agricultura & & 1 & 1 & $\mathbf{1 , 1 1}$ \\
\hline Seguros & 1 & & 1 & 1,11 \\
\hline Petróleo & & 1 & 1 & $\mathbf{1 , 1 1}$ \\
\hline Total general & $\mathbf{5 3}$ & $\mathbf{3 7}$ & $\mathbf{9 0}$ & \\
\hline
\end{tabular}

Fuente y elaboración propia.

De 37 organizaciones que mantienen un sistema de gestión de calidad, el 38\% (14) tienen un sistema simple y práctico y el 62\% (23) mantienen un sistema complicado. Si bien este artículo no representa el estudio de casos, con la información recolectada a lo largo de 12 años, los autores consideran que la diferencia radica en el enfoque de los diseñadores, implementadores y administradores de los sistemas de gestión, que en unos casos construyen modelos complicados, orientados al control y con alta carga administrativa, a pesar de que la actual versión de ISO flexibiliza, por ejemplo, la carga documental, y en otros casos se evidencian sistemas de gestión simples, prácticos y acordes con las dinámicas empresariales dominantes. 


\section{Bases teóricas de la productividad y el modelo Lean}

La productividad $^{5}$ es una importante métrica del desempeño para los países y las empresas; se relaciona con la eficiencia en los procesos, la producción y el uso de los recursos. La variación y el descenso en la productividad mundial en los últimos 70 años, desde tasas de crecimiento del $5 \%$ en 1950 hasta valores menores al $1 \%$ en 2015, son el reflejo de una crisis que crece. Para poner en contexto el impacto, según Yves Morieux (2015), con tasas de crecimiento menores al $1 \%$ se requerirían al menos tres generaciones para que una familia, una empresa o un país y sus ciudadanos tengan mayor desarrollo y accedan a mejores prestaciones o beneficios que su anterior generación.

Figura 2

Variación de la productividad mundial

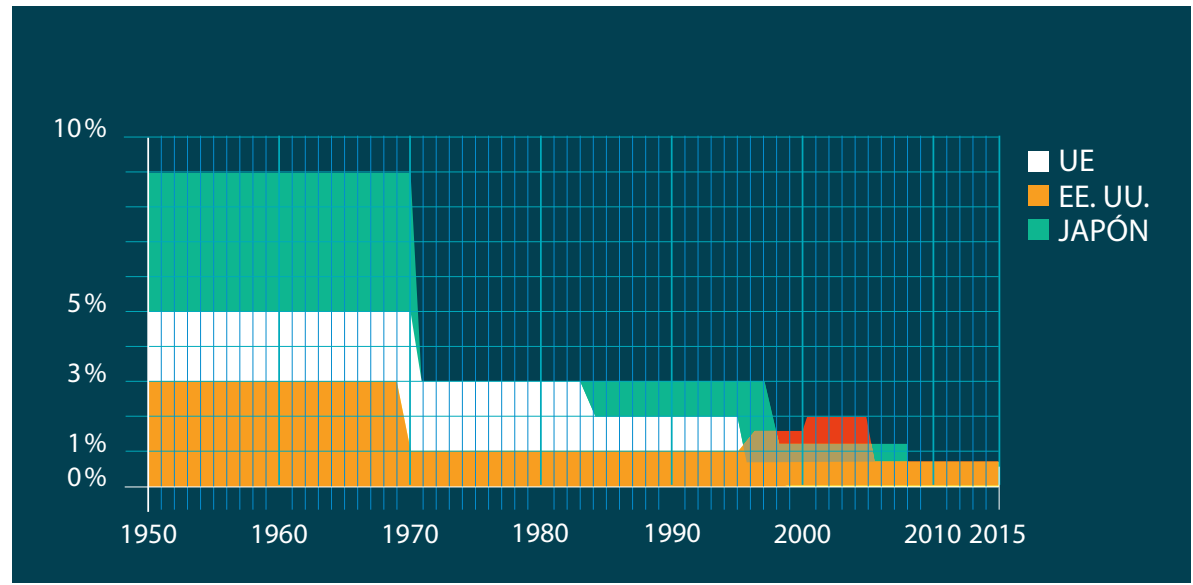

Fuente: Yves Morieux (2015).

5. "En cualquier contexto en el que se utilice la productividad, esta siempre es una comparación entre productos e insumos. Esta comparación puede realizarse en términos físicos o monetarios" (Medianero 2016, 24). 
Al analizar la productividad desde tres enfoques: la claridad (procesos), la responsabilidad (las personas y su autoridad) y las mediciones (indicadores), en cada uno de ellos los avances son representativos; además, con toda la tecnología que existe hoy, las causas del descenso de la productividad parecen encontrarse en las variables asociadas a la gestión, que se han incrementado en las organizaciones a tal punto que parecen estar en su contra porque cada vez son menos productivas y más complejas (Morieux 2015 min. 1-15).

Entre las teorías y modelos con mayor éxito probado en la era industrial se encuentra el método Toyota y los modelos Lean. ${ }^{6}$

La filosofía Lean Thinking-el pensamiento esbelto-nació a mediados de los años noventa con el lanzamiento del best seller The Machine that Changed the World: The Story of Lean Production (Womack, Jones y Roos 1990). Los principios de empuje de demanda (pull systems), just-in-time, calidad total, teoría de las restricciones, mejora continua y flexibilidad aplicados en la industria japonesa, más precisamente en Toyota, y conocidos como Toyota Way, han inspirado muchas industrias y metodologías (Medinilla 2012). Los principios fundamentales de esta filosofía son:

1. Comprender y maximizar el valor para el cliente: identificar todo lo que contribuye a mejorar la experiencia del cliente, es decir, pensar si el cliente pagaría por todo lo que hacen en la empresa o se deben eliminar informes, reuniones, reprocesos, sobreproducción u otros desperdicios.

2. Optimizar el flujo de valor: entender cómo las actividades de su empresa contribuyen al flujo de valor. Para determinar el flujo se debe identificar la relación con el cliente de principio a fin, e identificar cuánto tiempo toman las actividades, el costo, la secuencia, para saber el grado de valor que entrega al cliente.

3. La nueva economía: los nuevos productos se fabrican solo cuando el cliente necesita, reduciendo la necesidad de stocks, inventarios o exceso de producción. Hay que considerar la reducción de los ciclos de planificación, ejecución, liberación y feedback en períodos de pocas semanas.

6. A finales del siglo XIX surgió el primer pensamiento Lean Manufacturing en Japón por parte de Sakichi Toyoda, el fundador del Grupo Toyota. 
4. Flujo de pieza única: es la capacidad de una sola pieza de seguir el orden de flujo de manera ininterrumpida a través de todo el sistema, a la máxima velocidad posible.

5. Mejora continua Kaizen: es esforzarse por la perfección que procura hacernos mejores cada día.

Si bien el sistema de producción Toyota tiene ya más de medio siglo en empresas de producción industrial, apenas en los últimos 15 años otro tipo de industrias lo está aplicando en búsqueda de mejores resultados en su gestión. La industria del software es un ejemplo de ello. Desde 2002 desarrollaron cambios y crearon metodologías denominadas AGILES, ${ }^{7}$ utilizadas para resolver la complejidad de estrategias, proyectos y operaciones de desarrollo informático. En la actualidad este tipo de metodologías han capturado parte del mercado y se asocian con el éxito de empresas digitales como Apple, Amazon o Airbnb. Agilidad es un mindset, es una cultura de trabajo a través de un camino continuo de exploración, adaptación y aprendizaje, que busca generar valor al producto o servicio de forma evolutiva e incremental, basado en las personas. El enfoque esbelto ha demostrado que elimina las MUDA,${ }^{8}$ en definitiva, elimina variables que complejizan la organización y mejoran la productividad.

\section{La tecnología en los procesos}

Factor importante en el desarrollo organizacional de los últimos cinco decenios es la relación procesos-TIC. Los sistemas de información cambiaron de ser soluciones verticales orientadas al organigrama por soluciones transversales vinculadas al proceso. A continuación, representamos en línea de tiempo la relación entre las tecnologías asociadas a procesos.

7. Ejemplos de metodologías ágiles: scrum, design thinking, management 3.0, lean startup.

8. Los $M U D A$, término japonés que significa "inutilidad; ociosidad; superfluo; residuos; despilfarro. Existen siete elementos en la organización: tiempo, transporte, procesos, inventario, movimientos, defectos, sobreproducción (Menéndez 2014). 


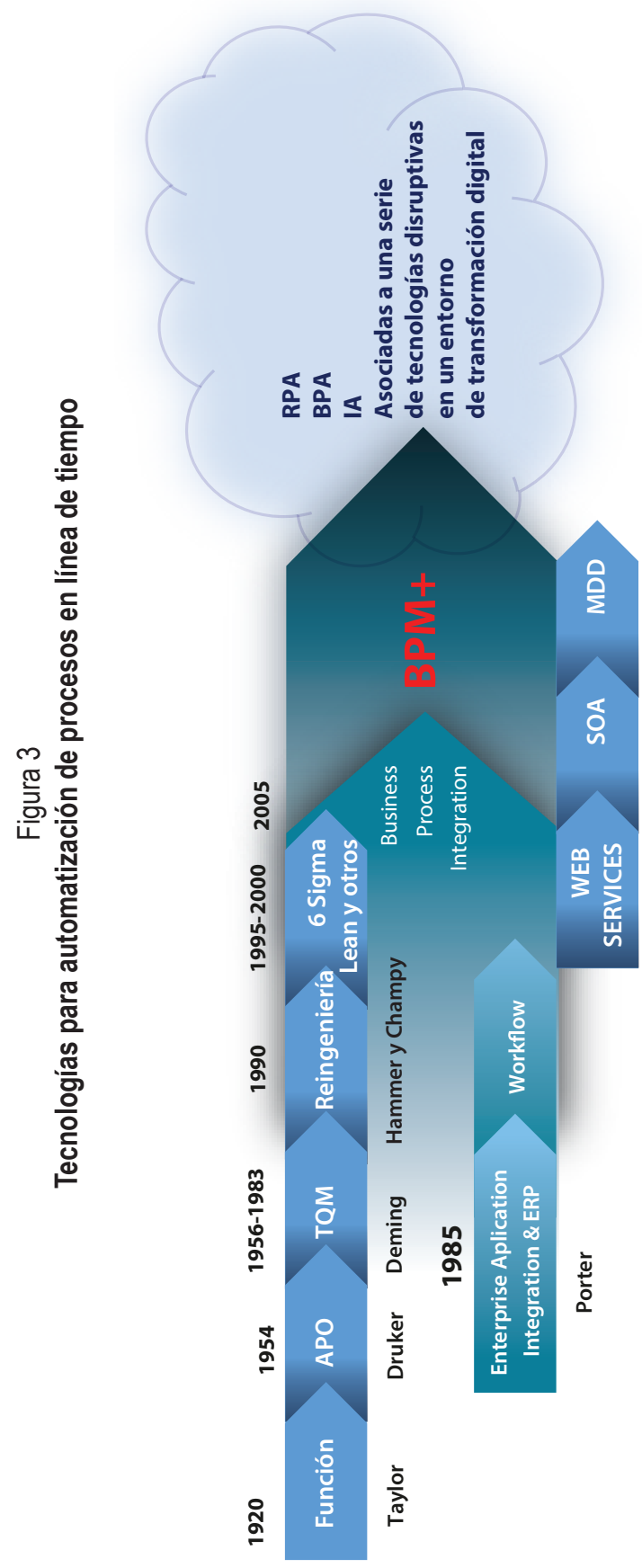

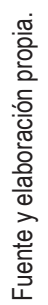


Los primeros sistemas integraron los procesos de la empresa, es el caso de los ERP (Enterprise Resourse Planning); en cambio, los workflows y BPMS (Business Process Management System) se diseñaron y automatizaron desde las actividades y a medida de las necesidades del flujo de proceso. En la última década apareció RPA (Robotic Process Automation), herramientas informáticas que se presentan como excelentes opciones para eliminar tareas repetitivas y manuales, que dejan de lado el enfoque sistémico de un flujo de proceso para concentrarse en actividades o tareas específicas.

Si bien estos sistemas han permitido flanquear la discrecionalidad de las personas en la ejecución del proceso, en la implementación los managers priorizaron el control de la ejecución, la eficiencia y finalmente la experiencia de usuario. Se han requerido al menos tres o cuatro versiones del proceso automatizado para explotar su potencial en un modelo digital. Es posible que el enfoque de los diseñadores de las soluciones informáticas esté enmarcado con las características de los modelos de procesos de la era industrial; si esto influye en la propuesta del proceso automatizado para la era digital, se generaría una distorsión similar a la expuesta al analizar los resultados de los sistemas de gestión de la calidad.

\section{Discusión}

Es evidente que los cambios de la cuarta revolución industrial impactan a los modelos de gestión, y la gestión por procesos no sería la excepción.

La gestión por procesos fue el centro del modelo de gestión de la tercera revolución industrial; la cuarta revolución industrial incluye tecnologías disruptivas y cambios en el enfoque que tiene como centro a las personas.

En las organizaciones digitales las tareas repetitivas y procedimentales serán automatizadas, sin importar cuán sofisticadas sean, quedarán fuera aquellas actividades de creación donde sea necesario el criterio humano por la complejidad de las interacciones o aquellas que impliquen una dimensión emocional. Las personas deberán redescubrir su rol e incluso su profesión, y probablemente varios puestos de trabajo serán reemplazados. 
Los diseñadores de procesos, soluciones informáticas, los managers y los propios ejecutores de la gestión deben asumir un desafío de cambio, analizar con pensamiento crítico las metodologías, buenas prácticas y herramientas que les permitan moverse del paradigma de eficiencia, eficacia y mejora continua característicos de la tercera revolución industrial hacia la innovación, adaptabilidad, flexibilidad, personalización, rapidez, prioridad y expansión acordes a la gestión en la era digital.

El desafío fundamental estará en las personas, quienes deberán adquirir conocimientos y habilidades para enfrentar la transformación que implica gestionar organizaciones digitales, en una sociedad hipervinculada, donde las interacciones complejas posiblemente sean la norma que haga de los procesos estructuras frágiles y cuyo reto principal sea mantener organizaciones humanizadas.

\section{Conclusiones}

Es necesario un cambio de enfoque para resolver la complejidad de la gestión en organizaciones digitales. Los modelos de la era industrial no responden al entorno VUCA. Empresas, universidades, centros de desarrollo, profesionales, y todos aquellos que estén involucrados en la gestión empresarial deben mirar al interior de las organizaciones con sinceridad y evaluar si no están situados en el concepto denominado superioridad racional, que trata los problemas actuales considerándose superior a dichos problemas, que cree que con los mismos procesos, personas, herramientas y modelos de gestión de siempre se resolverán los nuevos desafíos, aun cuando esto puede ser un fracaso anunciado.

En la era digital será necesario encontrar soluciones en un plano creativo, innovador y posiblemente original, donde la gestión por procesos reasigne el protagonismo que tuvo en la tercera revolución industrial para convertirse en un componente del vínculo que forman a personas y tecnología alineadas al propósito de la organización. 


\section{Referencias}

Gray, Sunni Brown, y James Macanufo. 2012. Gamestorming. Beijing: Deusto.

Hernández, Vizán. 2013. Lean Manufacturing. Conceptos, técnicas e implantación. Madrid: Fundación EOI.

Highsmith, Linda Luu, y David Robinson. 2020. EDGE: Value-Driven Digital Transformation. Londres: Pearson Education.

Hilbert, Martin. 2015. “¿Qué son los sistemas complejos?”. Accedido enero de 2020. https:// bit.ly/2AbSjD8.

Medianero, David. 2016. Productividad total: teoría y métodos de medición. Lima: Macro.

Medinilla, Ángel. 2012. Agile Management: Leadership in an Agile Environment. Berlín: Springer.

Menéndez, Gregorio. 2014. "Los 7 MUDAS: ¿sabes cuáles son los 7 desperdicios de las empresas?". Accedido diciembre de 2019. https://prevenblog.com/las-7-mudas/.

Ochoa, José. 2018. “¿Complicado o complejo?”. Video de YouTube, a partir de video conferencia. Accedido diciembre de 2019. https://www.youtube.com/watch?v=qbgmaB2_4f0\& $\mathrm{t}=10 \mathrm{~s}$.

Ochoa, José, y Juan Carlos Piñuela. 2017. Gestión y Proyectos. Quito: CODEU.

Schwab, Klaus. 2019. "La clave para entender el surgimiento de la cuarta revolución industrial". Dinero. Accedido diciembre del 2019. https://bit.ly/3eDGAfz.

Womack, Jones, y Daniel Roos. 1990. The Machine that Changed the World: The Story of Lean Production. Detroit: Free Press.

Yves, Morieux. 2014. "6 reglas para simplificar el trabajo, conforme se vuelve más complejo". Video de YouTube. Accedido noviembre de 2019. https://www.youtube.com/watch?v $=0 \mathrm{MD} 4 \mathrm{Ymjyc} 2 \mathrm{I} \& \mathrm{t}=540 \mathrm{~s}$.

---. 2015. "How Too Many Rules at Work Keep You from Getting Things Done". Video de YouTube. Accedido noviembre de 2019. https://www.youtube.com/watch?v=t_NoFstCmQ. 